\title{
Susceptibility/manifestations of different age groups with various comorbidities to COVID-19 infection
}

\author{
Albin John BA, Freedom Ha BS, Mimi Zumwalt MD
}

\begin{abstract}
The COVID-19 pandemic has taken a great toll on many families. From its rapid spread to debilitating outcomes, the virus has wreaked havoc on healthcare systems around the world. As researchers study this novel virus, the public continues to seek more information on who is the most susceptible and which population will be affected by the more severe manifestations of the disease. As a result, scientists have started analyzing the variable effects of COVID19 infection in different age groups. While the information is still nascent, these studies demonstrate that no one is immune, that all are susceptible to infection by this virus, and that certain demographics of the general population have more severe disease than others. This literature review examines how COVID-19 has affected different age groups, from neonates to older adults, by exploring statistics, mechanisms, and possible risk factors. This article will also investigate the role of comorbidities in increasing the severity of this viral infection.
\end{abstract}

Keywords: COVID-19, SARS-CoV-2, coronavirus, age, neonates, pregnancy, older adults, mechanism, comorbidities, angiotensin converting enzyme 2

\section{INTRODUCTION}

Different age groups historically have been shown to be differentially affected by infectious diseases. For example, during the Spanish flu of 1918, children were not significantly affected, while young adults were severely harmed. In the H1N1 influenza outbreak, the age span widened to include a population from the ages of 5-59 years old. Other infections, such as polio, presented less in neonates and more in adolescents. Across various diseases, children have been noted as being more resilient than other age groups. This may be due to the increased activation of their innate immune system and healthier respiratory tracts. ${ }^{1}$

During the SARS epidemic, individuals who were 25 years old or younger were less affected by the

Corresponding author: Mimi Zumwalt Contact Information: Mimi.Zumwalt@ttuhsc.edu DOI: $10.12746 /$ swrccc.v8i35.731 respiratory illness than those above the age of 60 . In fact, the mortality of infected patients rose to greater than $50 \%$ in older populations. This age-based susceptibility was also seen in murine models of the disease. Researchers noted that at younger ages (2 months old), mice were resistant to the virus (mouse adapted SARS-CoV, MA15). However, at 8-9 months, there was increased mortality, especially in male mice. This increased mortality demonstrated significant age (greatly affects older mice) and sex (greatly affects males) dose response. ${ }^{2}$

\section{Coronavirus}

Coronaviruses (CoV) are a group of RNA viruses that can affect both humans and animals. With spike projections that extend from the surface of the virus, coronaviruses have a corona or crown-like appearance. ${ }^{3}$ This family of RNA viruses has four major groups: $\alpha, \beta$, $\varepsilon$, and $\gamma$, the $\alpha$ and $\beta$ groups of coronaviruses can infect humans. ${ }^{4}$ The world has experienced the effects of this family of viruses, especially in the 
SARS-CoV (severe acute respiratory syndrome coronavirus) of 2003, a viral respiratory illness that caused severe respiratory tract infections. ${ }^{5}$ SARS-CoV replicated in the respiratory tract and within alveolar cells. It promoted an enhanced pro-inflammatory response that caused pulmonary damage due to increased inflammatory macrophages and neutrophils in the lungs. ${ }^{2,6}$ Patients who were severely affected by SARS had a rapid innate response and a dampened adaptive response; those who survived SARS had an increase in their adaptive response. ${ }^{7}$

\section{COVID-19}

The novel COVID-19 disease caused by the SARS-CoV-2 virus presents with many similarities with SARS-CoV of 2003. COVID-19 is hypothesized to have "jumped" from bats to humans at Wuhan's wet markets in the last quarter of $2019 .{ }^{4}$ With a basic regeneration number of 2.2, COVID-19 is an infectious disease with a long incubation period; it has strong human to human transmission and is spread through respiratory droplets from infected individuals. This virus appears to be more virulent in the lower respiratory tract. $^{1,8}$

SARS-CoV-2 is a single stranded RNA virus with a length of 29,903 base pairs. ${ }^{9}$ The genome has a large 5' open reading frame with 16 enzymes for viral replication. A third of the genome, at the 3 ' end, contains attachment and structural proteins, such as the spike, membrane, and nucleoproteins. There are many accessory proteins interspersed in this segment of the genome that have a role in subverting the host defense, in causing deleterious inflammation, and in stimulating apoptosis of host cells. ${ }^{7}$

The spike protein is a homotrimer glycoprotein that is integral to the virus's ability to get into cells. It has two functional units, S1 and S2, that act as a binding region and a binding regulator, respectively. Different regions on the $\mathrm{S} 1$ recognize different types of receptors. SARS-CoV-2 has a furin cleavage site between its S1 and S2 domains, which differentiates it from SARS-CoV and increases its potency. Its trimer has multiple different structures that can bind with receptors, thus adding to virulence. In contrast, other viruses in the family have only one closed trimer state. ${ }^{10}$

\section{MECHANISM OF INVASION/INFECTION}

To infect humans, the spike protein on SARS-CoV-2 particles binds to the angiotensin converting enzyme 2 (ACE2) receptor. The ACE2 receptors are found in mucosal cells exposed to air, such as the nose, eyes, and mouth. ${ }^{8}$ It is a carboxy peptidase that degrades angiotensin 2 made by angiotensin converting enzyme 1 (ACE1). ${ }^{11}$ Therefore, ACE1 and ACE2 are counterbalancing enzymes. ${ }^{12}$ Angiotensin 2 can cause fluid accumulation in the pulmonary alveoli due to its role in reducing clearance of fluid and can increase the death of endothelial cells, which, in turn, promotes vascular permeability. ${ }^{11}$

A type 1 membrane protein, ACE2 is bound by SARS-CoV-2 and downregulated. This downregulation increases angiotensin 2 production by ACE. ${ }^{5,6}$ Along with fluid, immune agents also enter the lungs due to increased vascular permeability. These natural inflammatory agents' invasion into lung tissue as an attempt to protect the host can cause lung damage and cell death. ${ }^{11}$ Figure 1 depicts the virus's mechanism of action.

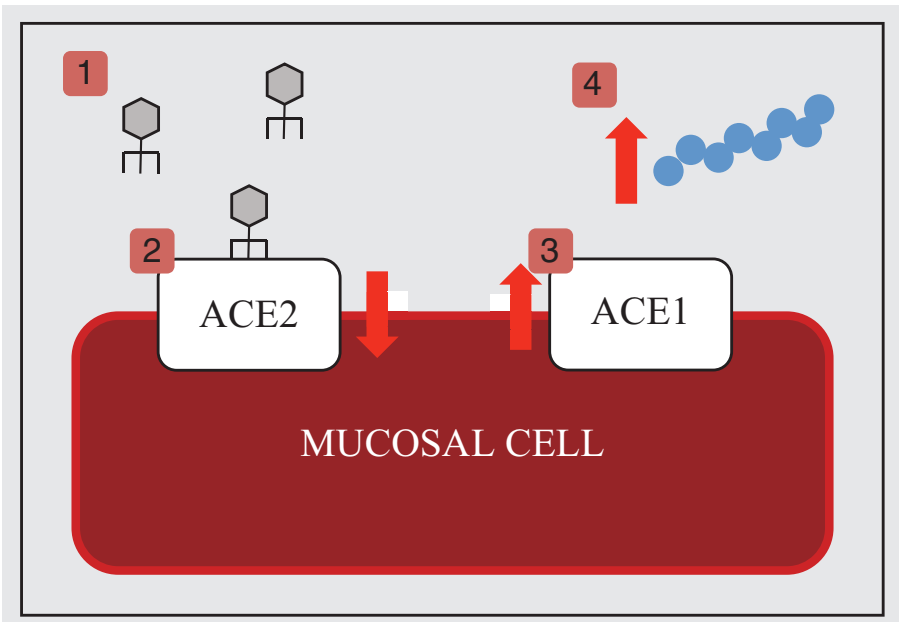

Figure 1. 1) The virus enters the lungs through respiratory droplets. 2) The ACE2 receptor is downregulated upon binding of the virus. The components of the virus enter the cell and undergo replication and maturation. 3) The downregulation of ACE2 increases the activity of ACE1.4) Increased ACE1 activity increases angiotensin 2 production Angiotensin 2 activates type 1a receptors and increases vascular permeability. 
In general, viral infections, such as SARS-CoV-2, can cause pulmonary damage and acute respiratory distress syndrome (ARDS) by creating a cytokine storm or Cytokine Release Syndrome (CRS) which presents like secondary hemophagocytic lymphohistiocytosis and can cause multiorgan failure. Many severe COVID-19 cases have a cytokine storm consisting of interleukins and TNF-alpha. ${ }^{6}$

After the virus binds to the ACE2 and enters the cell, its RNA is replicated. The spike protein, envelope, and membrane travel to the endoplasmic reticulum of the infected cell where the virus matures and is assembled to be pushed out via exocytosis. ${ }^{3}$

Coronaviruses are recognized by the toll-like receptor 7. The sensors, RIG1 and MDA-5, are activated by the cellular damage caused by the virus. The T-cells are primed when sensors react to viral peptides. Both T cells, CD8+ and CD4+, promote viral clearance by killing virions and mounting an antibody response. ${ }^{7}$ The antibody response from the host searches for and binds to the S2 domain of the spike protein. ${ }^{10}$ Both the CD8+ T cells and the neutrophils that migrate due to the chemoattractant IL-8 can cause extensive lung damage. ${ }^{13}$ The sensors also increase Type 1 interferon and cytokines. The interferons inhibit virus replication and are most effective in the early response process. If this is delayed, the same interferons can cause deleterious effects by damaging the host cells. ${ }^{7}$

Radiology can be used to diagnose and track the progression of COVID-19. Ground-glass opacities and notable septal thickening are seen in the images of COVID-19 patients. The right lower lobe is preferentially targeted; however, as the disease progresses, the patient eventually shows bilateral pulmonary damage. The ongoing disease progression in the lungs can precipitate acute respiratory distress syndrome (ARDS). ${ }^{14}$

In a study of 54 COVID-19 patients, investigators found that many criteria used for sepsis evaluation can also be applied to severe cases of COVID-19. Indicators, such as macrophage activation syndrome, low human leukocyte antigen $D$ related expression (HLA-DR), low numbers of CD4, CD19, and NK cells, were seen in 28 COVID-19 patients who had severe respiratory failure. The virus inhibits HLA-DR expression, a molecule on CD14 monocytes that serves as an indicator for immune dysregulation. The virus upregulates expression of IL-6 and TNF-alpha, which, in turn, downregulate host defenses, such as lymphocytes and HLA-DR expression. This dysregulation can be identified by increased levels of circulating ferritin. Reduction of HLA-DR expression correlates with the onset of severe respiratory dysfunction. Furthermore, those infected with COVID-19 had high levels TNFalpha and IL-6. In addition, IL-6 levels increased with disease severity and reduced HLA-DR expression and lymphocyte counts. ${ }^{7,15}$

Adverse outcomes correlated with age, comorbidities, and sex. Furthermore, if patients at the time of admission had increased D-dimer (greater than $1 \mu \mathrm{g} /$ $\mathrm{mL}$ ), they were more likely to have severe complications and eventually die. ${ }^{16}$ Patients who had severe cases of COVID-19 also had lymphopenia. ${ }^{13,16}$

While many symptoms and transmission methods may be similar, the patient population and latency of SARS-CoV-2 differ from SARS-CoV. First, COVID-19 has a broader susceptible population. People who have not been previously infected are all susceptible to this novel viral infection. Second, while SARS patients presented symptoms within 1 to 4 days, COVID-19 infections have a longer latency period, taking on average 3-7 days for symptoms to manifest. Furthermore, there is a higher male to female ratio (2.7:1) infection rate in COVID-19 that does not occur in the 2003 SARS $(1: 1.25) .{ }^{5}$ Third, COVID-19 virus binds to ACE2 more efficiently than SARS-CoV. ${ }^{5}$

This literature review will analyze how aging and comorbidities correlate with COVID-19 outcomes. By studying the similarities and differences in viral progression across ages/lifespan and by investigating associated medical conditions and their roles in increasing risk for adverse outcomes, this article will try to explain how COVID-19 infection affects different segments of the general population.

\section{COVID-19 INFECTION IN NEONATES}

Infants as young as 30 hours old have tested positive for COVID-19. ${ }^{8}$ While all children are susceptible 
to COVID-19, very young children, especially those under the age of 1 , have been critically ill from COVID19. ${ }^{4}$ The source of COVID-19 infection in neonates has not been extensively studied. Conclusive evidence of vertical transmission from mother to child has not yet been reported. ${ }^{17}$ Researchers searched for evidence of vertical transmission by looking at outcomes in pregnant women who contracted COVID-19 in the third trimester of pregnancy. Pregnant women can be more susceptible to respiratory infections since they are immunosuppressed. In addition, the symptoms of COVID-19 are not tolerated well in pregnant women. ${ }^{18}$

In a study of 9 pregnant women with no underlying medical conditions, 2 of the 9 patients experienced fetal distress but none (neither mother nor child) suffered any lasting or life-threatening problems. All these patients underwent caesarean deliveries because physicians were unclear whether there could be intrapartum transmission from mother to child during a vaginal delivery. COVID-19 was not found in any intrauterine samples, including the amniotic fluid and cord blood, or in the neonatal throat swab. The study postulates that if contracted in the third trimester, COVID-19 may not have vertical transmission to neonates. Vertical transmission during the first and second trimester has not been extensively studied. The researchers also noted that post-partum breast milk tested negative for COVID-19. Studies testing viral transmission via the vaginal mucosa have not yet been done. ${ }^{18}$

ACE2 is highly expressed in the placenta and in fetal organs, such as the fetal heart, liver, and lung. Components of the placenta, such as the cytotrophoblasts and the syncytiotrophoblast, and components of the decidua, all contain ACE2 receptors. The extravillous trophoblast, however, does not have receptors for ACE2. Researchers hypothesize that the expression of ACE2 at the placenta and decidua increase during pregnancy. These findings indicate that placenta and decidua can be infected by the virus. Furthermore, ACE2 expression in fetal organs, especially the neonatal lung, increases susceptibility of the baby to the virus. Even though previous studies have not shown any evidence for vertical transmission, the possibility does exist. Previous studies are limited because the data include only pregnant women who, in their third term, contracted the virus. The time from onset of illness to delivery was also very short. ${ }^{19}$ Some studies have also noted an increasing risk in preterm neonates and possible fetal distress when mothers are infected with COVID-19. ${ }^{20}$

\section{COVID-19 INFECTION IN CHILDREN}

As the pandemic unfolded, preliminary statistics gave a false belief that older children were immune to COVID-19. A Chinese study of 72,314 subjects noted only $0.9 \%$ of 44,672 confirmed COVID-19 cases involved children under the age of 10 years old. In Italy, only $1.2 \%$ of 22,512 COVID-19 cases were in young patients. ${ }^{21}$ By March 2020, however, German researchers noted that there was an increasing risk for children under the age of 6 for severe disease progression. As of April 6, 2020, there were 33 children in 21 German clinics who were hospitalized due to COVID19. In fact, $45 \%$ of these cases (15/33) were infants and newborns. ${ }^{22}$ As more new data are collected, the belief that children are immune to COVID-19 is incorrect. On a positive note, compared to adults, children are statistically less likely to suffer adverse outcomes due to COVID-19. Italian health officials noted that young adults and children in their country were not dying from COVID-19 infections. ${ }^{21}$

However, children are just as susceptible to this viral infection as other age groups. ${ }^{23}$ Children can also be more exposed to the virus because they lack the proper protective equipment. ${ }^{8}$ Furthermore, since the majority can't be advocates for their own health and due to the lack of asymptomatic testing, the spread of COVID-19 in children may be greatly underreported. ${ }^{8,17}$ Pediatric patients, with their developing immune systems, can be very susceptible to upper respiratory tract infections in general. ${ }^{24}$

From a study of 2,135 pediatric patients (with only 1 child mortality), researchers have shown that pediatric patients, while susceptible, are less likely to have adverse outcomes from the virus than other age groups. ${ }^{4}$ Respiratory manifestations of COVID19 infection are less severe in children than in adults. They can present with the symptoms, such as difficulty breathing, fever, and a persistent cough, but most cases were largely asymptomatic or had mild symptoms. ${ }^{21}$ In addition, children tend to recover 
within 1 to 2 weeks of onset even if there is increased disease severity and progression. ${ }^{23,25}$

The difficulty with identifying COVID-19 in pediatric patients is differentiating it from other respiratory illnesses found in these patients. Most young patients studied had been co-infected with other organisms. Radiology and blood work can characterize viral progression in pediatric patients. In pediatric computed tomography (CT), subpleural infiltrates and ground glass opacities were noted, and consolidation was seen as the disease progressed. When the patient was severely affected by the virus, the infiltrates and consolidations were found diffusely in both lungs. As patients recovered, the ground glass opacities and consolidations slowly decreased and could be completely reabsorbed in some patients. ${ }^{24}$ Both children and adults show a similar progression in CT scans. Children have normal C-reactive protein, ALT, and myocardial enzyme levels on lab tests. ${ }^{9}$

Children may also have a longer incubation period after being infected by COVID-19. The average incubation period in children is almost 1 day longer than in adults ( 6.5 days vs. 5.4 days). In children, intestinal survival of the virus can be quite long. For example, anal swabs tested positive for the virus in children for up to 51 days. ${ }^{8}$ As a result, children may increase the spread of this viral infection with their close contacts due to the largely asymptomatic nature of the disease and the extended incubation period. . $^{8,17}$

Immunocompromised children and children with prior lung disease, such as a previous respiratory tract infection, had a more difficult time controlling the infection. ${ }^{17,21}$ For example, children with juvenile idiopathic arthritis are immunocompromised and thus are more susceptible to infections in general. ${ }^{3}$

\section{COVID-19 INFECTION IN ADULTS}

As of February 2020, mortality in adults over the age of 80 has been reported by the WHO-China fact finding mission at approximately $21.9 \%$. Other studies from China have placed patients above the age of 80 at having a mortality of $14.5 \%$. In the United States, the fatality rate for adults over the age of 85 was similarly high $(10 \%-27 \%){ }^{26}$ The majority $(80 \%)$ of American deaths due to COVID-19 has occurred in the older populations ( $>65$ years of age). ${ }^{7}$ The rate of hospitalization in older patients has increased during March 2020 to 17.2 adults for every 100,000 Americans. ${ }^{27}$

Older adults, especially those in nursing homes, are the most affected by the virus due to advanced age, increased viral spread risk, and difficulty in early recognition of symptoms. Older patients often have compromised immune systems due to pre-existing conditions, such as hypertension, diabetes, and renal and respiratory diseases. In general, as one ages, there is an increase in comorbidities and underlying conditions. These medical comorbidities tax the immune system making the body more susceptible to infection and serious complications when infected. The CDC states that $63.1 \%$ of adults in the U.S. have hypertension, $38.0 \%$ have chronic kidney disease, and $26.8 \%$ have diabetes. ${ }^{26}$ In a study of 191 patients from the Wuhan and Jinyintan Hospitals, the median age was 56 years old and most were males. Around $50 \%$ of patients had underlying conditions, such as hypertension. Sepsis was the most common complication seen with adverse COVID-19 outcomes. Other complications included ARDS and systemic shock. Ten out of 32 patients who had to be intubated and did not survive were infected by a ventilator-associated pneumonia. ${ }^{16}$

Another recent study that surveyed 1,482 patients with confirmed COVID-19 during March 2020 noted that $74.5 \%$ of patients were older than 50 . A large proportion of these (89.3\%) had one or more medical comorbidities. Hypertension at $49.7 \%$ was the leading underlying condition in patients with COVID-19. Obesity and chronic pulmonary disease follow closely at $48.3 \%$ and $34.6 \%$, respectively. Other associated risk factors included diabetes and cardiovascular disease ${ }^{27}$ Pulmonary fibrosis (PF) can be a component of lung tissue consolidation that leads to ARDS in COVID-19. Pulmonary fibrosis has developed in the lungs of COVID-19 patients, and there is a positive correlation between disease duration and extent of fibrosis. Angiotensin-converting enzyme 2 (ACE2) expression also directly correlates with PF, and cells that express ACE2 appear to contribute to PF development. The likelihood of PF increases with age and tends to be higher in males. ${ }^{28}$

Medications used in comorbidities can have a significant role in changing the body's response to 
COVID-19. ACE2 expression is increased in diabetic patients (types 1 and 2), especially in patients on ACE inhibitors (ACEI) and angiotensin II blockers (ARBs). Similarly, hypertension is treated with ACE inhibitors and ARBs. Increased ACE2 could increase susceptibility to COVID-19. ${ }^{29}$ However, use of ACEI and ARBs can provide a protective role from adverse outcomes by inhibiting the renin-angiotensin system (RAS). The inhibition by ACEI and ARBs reduces angiotensin 2 production, thus decreasing the inflammatory cytokine activity. Researchers have noted improved outcomes in a small study of 417 COVID-19 patients in Shenzen Third People's Hospital taking these antihypertensive medications. However, the small size of this study limits global validity of this finding and more research into ACEI and ARBs must be done in larger populations. ${ }^{30}$

Nonsteroidal anti-inflammatory drugs (NSAIDs) increase ACE2 expression in diabetic rats. Therefore, it has been suggested NSAIDs increase ACE2 and increase susceptibility and mask symptoms of COVID19 , such as fever, that could help with early diagnosis. ${ }^{6}$ Corticosteroids (CS) inhibit the patient's immune system during responses to pathogens and also delay infection clearance. ${ }^{6}$ This class of anti-rheumatic drugs makes patients more vulnerable to infection from other diseases. It has been shown that patients who receive prednisone, an immunosuppressant and TNF inhibitor, were at a higher risk for infections. Similarly, the British Society for Pediatric and Adolescent Rheumatology also noted that methotrexate users had a higher incidence of infection. ${ }^{3}$ As a result, rheumatoid patients are particularly susceptible to infectious disease due to their medication regimen, and because viral infections (even though more commonly bacterial infections) can cause flare ups of their autoimmune disease. ${ }^{6}$

However, it may be too soon to conclude that immunosuppression leads directly to severe outcomes. In a systematic review of immunosuppressed patients, researchers noted that patients who were immunosuppressed made up a small portion of the overall COVID-19 cases and may have better outcomes than patients with other comorbidities seen in most COVID patients. This study combined a variety of sources, from case series to retrospective studies, and collected data on 110 patients, mostly from China. Most of these were cancer patients; the rest of the patients were either immunodeficient or post-transplantation recipients. Results showed that $20.9 \%$ (23) of the patients died, $8.2 \%$ (9) reached composite outcome of death or ICU admission, and $65.5 \%$ (72) of the 110 immunosuppressed COVID-19 positive patients did not require ICU beds but were admitted to the inpatient service/wards or discharged from the hospital. ${ }^{31}$

Preliminary data show that COVID-19 positive pediatric and adult cancer patients, transplant patients, and immunosuppressed patients do not seem to follow the pattern that infectious disease outbreaks negatively affect immunocompromised patients more than normal patients. Their immune status does not necessarily correlate with a poor prognosis or increased morbidity and mortality based on the current data from MERS-CoV, SARS-CoV, and SARS-CoV-2 infections. ${ }^{31}$ Although cancer is considered the most severe of the immunosuppressed diseases (transplantation, immunodeficiency from other conditions), the prognosis was not as poor as expected. Cancer patients tend to be older and immunosuppressed, but other risk factors, such as obesity, DM, cardiovascular disease, male sex, and age in combination seem, to present more risk than immunosuppression alone. These results, however, are limited by a small sample size, low number of pediatric patients (only 4), and an unequal sampling of those having immunocompromised conditions (i.e., cancer vs post-transplantation). ${ }^{31}$

Other risk factors contributing to COVID-19 infection include close proximity living quarters that can add to the viral spread. For older adults living in nursing homes, the large number of staffing personnel and frequent visitors can also increase the incidence of the virus infecting nursing home residents/ staff. ${ }^{32}$ French researchers have postulated that the basic regeneration number $\left(R_{0}\right.$ value) may be higher in these facilities due to the combination of compromised immunity and close inhabiting quarters. ${ }^{33}$

Furthermore, infectious symptoms may present atypically or may be difficult to distinguish and recognize early in elderly patients. ${ }^{7}$ Older adults do present with fever, cough, pain, and difficulty breathing. However, these are similar symptoms that are already common in the elderly due to pre-existing conditions. Over time, those patients who progress into the critical 
stages of COVID-19 have shown a greater likelihood of organ damage, such as kidney or lung injury, causing acute respiratory distress. Chest x-rays of COVID-19 of older patients demonstrate ground glass opacities and consolidations with bilateral pulmonary distribution. ${ }^{26}$

In King County in Washington state, $57 \%$ of residents $(13 / 23)$ in a nursing home tested positive for COVID-19. Of these infected patients, $77 \%(10 / 13)$ developed symptoms. In Seattle, 101 nursing home residents tested positive for COVID-19, and 33.7\% died. The Morbidity and Mortality Weekly report noted an almost doubling in ICU admission and Case Fatality Rates (CFR) in older adults in over just 1 month this year (February 12-March 16). Older patients who become symptomatic are more likely to require admission to the hospital and even to the ICU than younger adults. In addition, older adults are also more likely to develop ARDS than younger patients. ${ }^{32}$

\section{COVID-19 INFECTION-MECHANISTIC DIFFERENCES ACROSS AGE GROUPS}

Research has shown a possible difference in ACE2 expression across different ages. Some investigators have noted that ACE2 has a spike in its expression in days $1-3$ of life. ${ }^{19}$ Other researchers have seen similar trends in mouse models. Studies in mice also demonstrate a decrease in expression until adolescence, after which ACE2 is expressed again. This can be a possible explanation for the higher susceptibility to COVID-19 in neonates as compared to older children. ${ }^{13}$ However, not all literature sources agree on the age-dependent expression of ACE and ACE2. ${ }^{12}$

Besides ACE2 expression, the neonate's immune system is still immature, and this can contribute to increased COVID-19 susceptibility. When a baby is born, there is a reduced CD8+ T cell count. As the cytotoxicity of CD8+ cells are important in virus clearance, neonates, who have fewer cytotoxic CD8+ cells, are more likely to be hospitalized due to COVID-19 infection. ${ }^{13}$ In addition, the level of IL-6 is lower in neonates than in any other age groups, and they have a high quantity of ICAM-1, an adhesion molecule that promotes neutrophil migration to the lungs. However, when compared to adults, neonates have much lower numbers of neutrophils due to their nascent immunity pool. In a study of 92 patients with ARDS, neonates were more likely to have severe disease than other age groups. However, while these neonatal patients were the most susceptible, they had a lower death rate (older adults had the highest mortality). ${ }^{12}$

There are a few hypotheses to explain why older children may have less severe infections with COVID19 than other age groups. One interesting theory is that the binding of COVID-19 to the ACE2 in children may be reduced, possibly due to immature ACE2 receptors. ${ }^{4}$ The ACE2 has greater expression in welldifferentiated ciliated epithelial cells. Children have less differentiated cells than adults. Therefore, younger patients tend to have reduced ACE2 expression compared to older patients. ${ }^{13}$ Another postulation points to the heightened immune state that children possess since they are continuously exposed to new infections. As a result, their young bodies might have better host defenses against COVID-19 infection., ${ }^{4,13}$

As one gets older, the immune system ages with the body such that it clears viruses more slowly (Figure 2). People over the age of 60, even without comorbidities, have an almost 25 times increased risk of mortality

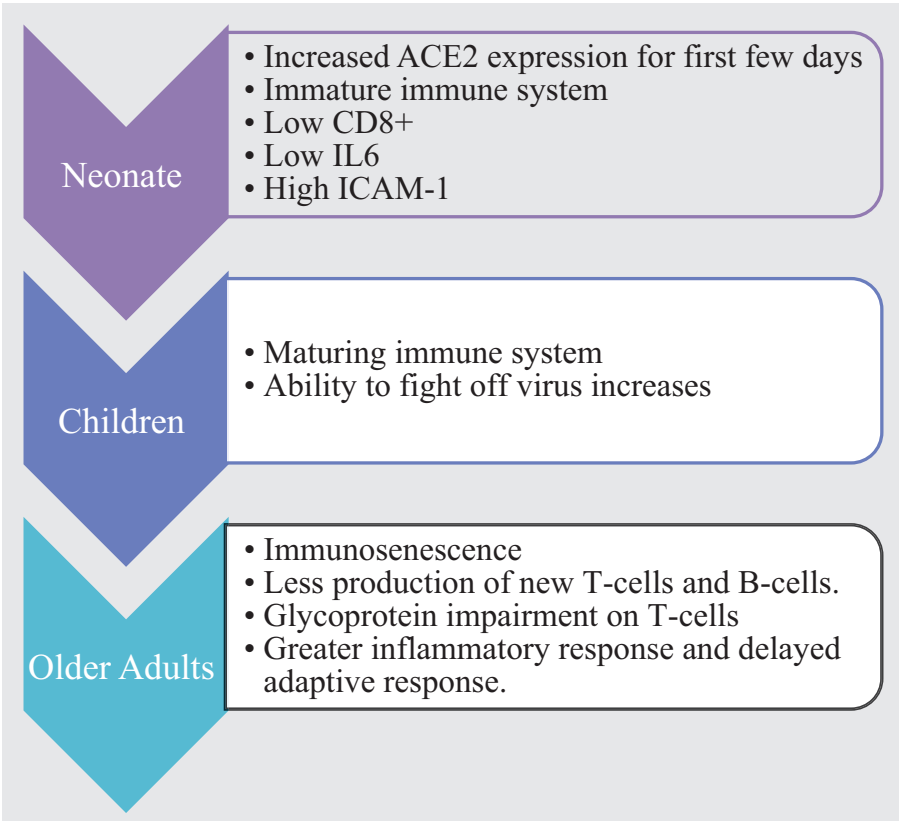

Figure 2. Maturation of the immune system. 
than younger adults. Furthermore, this mortality risk increases with underlying conditions, such as cancer and pneumonia. As the thymus involutes with aging and ceases in its immunogenerative role, T-cell and subsequent memory cell generation in turn decreases. This subsequently reduces the efficacy of vaccines in older patients. The change in immunity is named immunosenescence and results in a poor response to new antigens and increased inflammatory and autoimmune reactions to invading pathogens. ${ }^{34}$

Furthermore, with aging there is a decrease in the ability to make new cells from the bone marrow. Such an age related change impairs the adaptive immune response due to a reduction in the generation of $T$ and $B$ cells. ${ }^{35}$ As one gets older, there is a shift toward maintenance of existing T-cell diversity and reduced capacity to make new disease specific T-cells. ${ }^{13}$ This decrease in diversity also occurs in the B cell pool as there are fewer nascent $B$ cells and more disease exposed B cells. ${ }^{35}$ From a decrease in both new T-cell and $B$ cell production, to an age related decline in maintenance of T-cells by lymph nodes, aging reduces the ability of a coordinated defense against pathogens in the older body.

Glycoproteins on the surface of T-cells also may be reduced as one gets older, which leads to reduced function of these T-cells. In addition, CD4+ cells also lose their ability to control their inflammatory response to stimulation. The inability to discern correct induction of specific genes, such as STAT1 and not SHP1 , reduces their ability to mount an adaptive immune response since they spend less time in secondary lymphoid organs after exposure to an antigen. As patients age, their inflammatory response becomes paradoxically deleterious as it is relied upon more heavily to clear pathogens. Thus, the IL-6 levels become very high. Overall, older patients, in their natural reaction to pathogens, have extended innate responses and delayed adaptive responses. . $^{7,35}$

In addition, older patients have delayed interferon surges. While it is also important to note that coronaviruses have adapted to delay the surge of interferon, other markers in adult COVID-19 patients show the vulnerability of an aged immune system. For example, there is an increase in NK group 2 member $A$, which is a marker for T-cell exhaustion. This exhausted state reduces the response to infection, thus allowing for the disease to further propagate. ${ }^{13}$

Other factors contributing to the increased susceptibility of the aged body to infection include associated medical diseases. Therefore, the immune system becomes greatly taxed by the increasing comorbidities as one gets older. Of those who have medical conditions, $45.4 \%$ of patients who contract COVID-19 are at risk for serious complications. As patients age, the likelihood of serious complications rises to $80.7 \%$. The case fatality rate in China for adults with medical comorbidities was much higher than for those without. As the number of chronic conditions increases, the potential for grave complications rise as well. The Centers for Disease Control and Prevention has identified smoking, obesity, diabetes mellitus, hypertension, high cholesterol, and cancer as chronic comorbidities that can contribute to a higher risk for complications. ${ }^{36}$

Patients with underlying medical conditions, such as hypertension and diabetes, already have an overactive renin-angiotensin system (RAS) that can be amplified by the virus. ${ }^{11}$ The RAS system is made up of multiple components that help maintain homeostasis. Specifically, ACE/ATII/AT1R promotes vasoconstriction, inflammation, and fibrosis. In contrast, ACE2/ Ang1-7/Mas promotes vasodilation, anti-inflammation, and anti-fibrosis. ${ }^{37}$ The dysregulation of the RAS system caused by COVID-19 can set off an extreme inflammatory response that can affect multiple organ systems. As such, ACE2 expression due to comorbidities also can result in an increased susceptibility to COVID-19. The ACE2 level is high in the adipocytes of diabetics and obese patients, which could increase the risk of COVID-19 infection. Similarly, adiponectin levels are higher in those with less body fat mass and is negatively correlated with COVID-19 severity (less severe manifestations). The relationship between RAS and COVID-19 is an area for further study as more information becomes available. ${ }^{28}$

\section{LIMITATIONS}

This review is limited by the current available scientific literature and by shifting conclusions as a result 
of newer data. As an emerging virus, COVID-19 infection evidence is still being collected and published. With the small sample sizes included in English publications, common themes seem to appear but are not entirely conclusive. Furthermore, as additional clinical cases emerge, researchers are finding different, sometimes conflicting, information. As a result, this area of study is still developing and needs more time for data to develop for conclusions to be definitively drawn.

\section{Conclusions}

The COVID-19 pandemic has brought many nations and health care systems to their breaking points. From the rapid viral spread to the difficulty in controlling the virus, COVID-19 has greatly impacted all populations in the world. As it is still unfolding, researchers are trying to gather as much data as possible to explain this novel virus's potency and to understand how to protect the public since no one is immune. This review has found that all ages of the population are susceptible to the virus; however, an aging and/or compromised immune system increases the likelihood of an adverse outcome.

Article citation: John A, Ha F, Zumwalt M. Susceptibility/ manifestations of different age groups with various comorbidities to COVID-19 infection. Southwest Respiratory and Critical Care Chronicles 2020; 8(35):7-16

From: Department of Orthopedics Texas Tech University Health Sciences Center, Lubbock, Texas

Submitted: $5 / 14 / 2020$

Accepted: $7 / 5 / 2020$

Reviewer: Kenneth Nugent MD

Conflicts of interest: none

This work is licensed under a Creative Commons Attribution-ShareAlike 4.0 International License.

\section{REFERENCES}

1. Lee PI, Hu YL, Chen PY, et al. Are children less susceptible to COVID-19? J Microbiol Immunol Infect 2020.

2. Channappanavar R, Fett C, Mack M, et al. Sex-based differences in susceptibility to Severe Acute Respiratory
Syndrome Coronavirus infection. J Immunol 2017;198(10): 4046-53.

3. Licciardi F, Giani T, Baldini L, et al. COVID-19 and what pediatric rheumatologists should know: a review from a highly affected country. Pediatr Rheumatol Online J 2020; 18(1):35.

4. Dong Y, Mo X, Hu Y, et al. Epidemiology of COVID-19 among children in china. Pediatrics 2020.

5. Xu J, Zhao S, Teng T, et al. Systematic comparison of two animal-to-human transmitted human coronaviruses: SARSCoV-2 and SARS-CoV. Viruses. 2020;12(2).

6. Favalli EG, Ingegnoli F, De Lucia O, et al. COVID-19 infection and rheumatoid arthritis: faraway, so close! Autoimmun Rev 2020;19(5):102523.

7. Nikolich-Zugich J, Knox KS, Rios CT, et al. SARS-CoV-2 and COVID-19 in older adults: what we may expect regarding pathogenesis, immune responses, and outcomes. Geroscience 2020.

8. She J, Liu L, Liu W. COVID-19 epidemic: disease characteristics in children. J Med Virol 2020.

9. Lu Q, Shi Y. Coronavirus disease (COVID-19) and neonate: what neonatologists need to know. J Med Virol 2020.

10. Walls AC, Park YJ, Tortorici MA, et al. Structure, function, and antigenicity of the SARS-CoV-2 spike glycoprotein. Cell 2020;181(2):281-92. e6.

11. Sriram K, Insel PA. A hypothesis for pathobiology and treatment of COVID-19: the centrality of ACE1/ACE2 imbalance. Br J Pharmacol 2020.

12. Schouten LR, van Kaam AH, Kohse F, et al. Age-dependent differences in pulmonary host responses in ARDS: a prospective observational cohort study. Ann Intensive Care 2019; 9(1):55.

13. Yuki K, Fujiogi M, Koutsogiannaki S. COVID-19 pathophysiology: a review. Clin Immunol 2020:108427.

14. Shi $H$, Han $X$, Jiang N, et al. Radiological findings from 81 patients with COVID-19 pneumonia in Wuhan, China: a descriptive study. Lancet Infect 2020;20(4):425-34.

15. Giamarellos-Bourboulis EJ, Netea MG, Rovina $N$, et al. Complex immune dysregulation in COVID-19 patients with severe respiratory failure. Cell Host Microbe 2020.

16. Zhou F, Yu T, Du R, et al. Clinical course and risk factors for mortality of adult inpatients with COVID-19 in Wuhan, China: a retrospective cohort study. Lancet. 2020;395(10229): 1054-62.

17. Cruz AT, Zeichner SL. COVID-19 in children: initial characterization of the pediatric disease. Pediatrics 2020.

18. Chen H, Guo J, Wang C, et al. Clinical characteristics and intrauterine vertical transmission potential of COVID-19 infection in nine pregnant women: a retrospective review of medical records. Lancet 2020;395(10226):809-15. 
19. Li M, Chen L, Zhang J, et al. The SARS-CoV-2 receptor ACE2 expression of maternal-fetal interface and fetal organs by single-cell transcriptome study. PLoS One 2020;15(4): e0230295.

20. Morand A, Fabre A, Minodier P, et al. COVID-19 virus and children: what do we know? Arch Pediatr 2020;27(3):117-8.

21. Ludvigsson JF. Systematic review of COVID-19 in children shows milder cases and a better prognosis than adults. Acta Paediatr 2020.

22. Streng A, Hartmann K, Armann J, et al. [COVID-19 in hospitalized children and adolescents]. Monatsschr Kinderheilkd 2020:1-12.

23. Castagnoli R, Votto M, Licari A, et al. Severe Acute Respiratory Syndrome Coronavirus 2 (SARS-CoV-2) infection in children and adolescents: a systematic review. JAMA Pediatr. 2020.

24. Xia W, Shao J, Guo Y, et al. Clinical and CT features in pediatric patients with COVID-19 infection: different points from adults. Pediatr Pulmonol 2020;55(5):1169-74.

25. Hong H, Wang Y, Chung HT, et al. Clinical characteristics of novel coronavirus disease 2019 (COVID-19) in newborns, infants and children. Pediatr Neonatol. 2020;61(2): $131-2$.

26. Shahid Z, Kalayanamitra R, McClafferty B, et al. COVID-19 and older adults: what we know. J Am Geriatr Soc 2020.

27. Garg S, Kim L, Whitaker M, et al. Hospitalization rates and characteristics of patients hospitalized with laboratoryconfirmed coronavirus disease 2019-COVID-NET, 14 States, March 1-30, 2020. MMWR Morb Mortal Wkly Rep 2020;69(15):458-64.
28. Kruglikov IL, Scherer PE. The role of adipocytes and adipocyte-like cells in the severity of COVID-19 infections. Obesity (Silver Spring). 2020.

29. Fang L, Karakiulakis G, Roth M. Are patients with hypertension and diabetes mellitus at increased risk for COVID-19 infection? Lancet Respir Med 2020;8(4): e21.

30. Meng J, Xiao G, Zhang J, et al. Renin-angiotensin system inhibitors improve the clinical outcomes of COVID-19 patients with hypertension. Emerg Microbes Infect 2020;9(1):757-60.

31. Minotti C, Tirelli F, Barbieri E, et al. How is immunosuppressive status affecting children and adults in SARS-CoV-2 infection? A systematic review. J Infect 2020.

32. Mills JP, Kaye KS, Mody L. COVID-19 in older adults: clinical, psychosocial, and public health considerations. JCI Insight. 2020.

33. Etard JF, Vanhems P, Atlani-Duault L, et al. Potential lethal outbreak of coronavirus disease (COVID-19) among the elderly in retirement homes and long-term facilities, France, March 2020. Euro Surveill 2020;25(15).

34. Accardi G, Caruso C. Immune-inflammatory responses in the elderly: an update. Immun Ageing 2018;15:11.

35. Sadighi Akha AA. Aging and the immune system: An overview. J Immunol Methods 2018;463:21-6.

36. Adams ML, Katz DL, Grandpre J. Population-based estimates of chronic conditions affecting risk for complications from coronavirus disease, United States. Emerg Infect Dis 2020;26(8).

37. D'Ardes D, Boccatonda A, Rossi I, et al. COVID-19 and RAS: unravelling an unclear Relationship. Int J Mol Sci 2020; 21(8). 